Samantha J. Mills

Calvin Soh

James P. B. O'Connor

Chris J. Rose

Giovanni A. Buonaccorsi

Susan Cheung

Sha Zhao

Geoff J. M. Parker

Alan Jackson

\title{
Tumour enhancing fraction (EnF) in glioma: relationship to tumour grade
}

Received: 18 June 2008

Revised: 6 November 2008

Accepted: 25 November 2008

Published online: 7 February 2009

(C) The Author(s) 2009. This article is published with open access at

Springerlink.com
S. J. Mills $(\varangle)$ - J. P. B. O’Connor ·

C. J. Rose - G. A. Buonaccorsi •

S. Cheung $\cdot$ S. Zhao $\cdot$ G. J. M. Parker

A. Jackson

Wolfson Molecular Imaging Centre, Imaging Science and Biomedical

Engineering, University of Manchester,

27 Palatine Road, Withington,

Manchester, M20 3LJ, UK

e-mail: samantha.mills@

manchester.ac.uk

Tel: : 44-161-2750040

Fax: +44-161-2750030

S. J. Mills · C. Soh · A. Jackson

Department of Neuroradiology,

Hope Hospital,

Stott Lane,

Salford, M6 8HD, UK
Abstract The aim of this research was to determine whether the proportion of a tumour that enhances (enhancing fraction, EnF) and changes in EnF with enhancement threshold differ between low and high grade glioma. Forty-four patients (45 gliomas comprising 16 grade II, 5 grade III and 24 grade IV) were studied. Imaging included pre- and post-contrast-enhanced $\mathrm{T}_{1}$-weighted sequences and $\mathrm{T}_{1}$-weighted DCE-MRI. Thresholded enhancement maps were generated for each tumour by using a range of values of the initial area under the contrast concentration curve (IAUC). A plot of EnF versus threshold value was generated. We examined the relationship between tumour grade and enhancement metrics including: EnF (threshold IAUC $>0 \mathrm{mMol} \mathrm{s}$ ), EnF (threshold IAUC $>2.5 \mathrm{mMol} \mathrm{s}$ ), initial slope of the EnF/threshold curve $(\partial \mathrm{EnF}), \mathrm{IAUC}$, and two previously described signal-intensitybased metrics. EnF, defined as the proportion of tumour showing any enhancement (threshold IAUC> $0 \mathrm{mMol} \mathrm{s}$ ), showed no difference between low and high grade glioma. All other measures demonstrated significant differences between grade II and IV, and low (grade II) and high grade (grades III/ IV) gliomas ( $p<$ 0.01 ). Two measures, $\partial \mathrm{EnF}$ and Pronin's measure of enhancement, showed differences between grade III and IV $(p<0.05)$. No measure separated grade II from III. Metrics which describe the enhancing fraction and its variation with enhancement threshold $\partial$ EnF show considerably different behaviour in low and high grade tumours. These observations suggest that these metrics may provide important biological information concerning tumour biology and therapeutic responses and encourage further research to characterise and validate these novel biomarkers.

Keywords Glioma - Enhancement . DCE-MRI $\cdot$ Enhancing fraction

\section{Introduction}

Glioblastoma multiforme (GBM) is one of the commonest, aggressive and vascular primary brain tumours in adults. The tumours are structurally heterogenous, characterised by varying degrees of hypercellularity, cytoplasmic and nuclear pleopmorphism, mitoses and endothelial proliferation. Microvascular proliferation is a prominent feature and most marked in areas of necrosis and at the tumour-brain interface [1]. The marked spatial heterogeneity of histological features is accompanied by regional variations in vascular development and maturity, vascular density, blood flow, oxygenation, $\mathrm{pH}$, and interstitial pressure. Some effects of this heterogeneity are well known; for example, areas of hypoperfusion and hypoxia are associated with poor chemotherapy delivery and poor radiotherapy response [2].

The proportion of a tumour which is perfused, expressed as the enhancing fraction (EnF), has been proposed as a simple measure of bulk tumour perfusion [3, 4]. EnF has been shown to predict outcome to first-line chemotherapy in 
patients with advanced ovarian cancer [5] and to change rapidly in response to drug-induced variations in tumour interstitial pressure [3]. EnF is a potentially interesting metric since it differs from conventional measurements of microvascular structure and function in a number of potentially important ways. Firstly, conventional microvascular parameters such as regional blood volume (CBV) are highly heterogeneous in glial tumours and mean or median values are by definition unrepresentative [6]. Many research studies select the region of the tumour in which to make these measurements so that, for instance, the wellestablished relationship between $\mathrm{CBV}$ and tumour grade is based on some reports which have deliberately selected the highest areas of CBV [7] and others which report average values from all enhancing tumour tissue $[8,9]$. In comparison, EnF describes the perfusion characteristics of the tumour as a whole and will be significantly affected by regional variations in perfusion pressure and by global intracranial pressure. Secondly, $\mathrm{EnF}$ is a model-free parameter which, if clinically useful, will be easy to implement in a clinical environment, thus offering a simple alternative to parametric modelling approaches.

Measurement of EnF requires the classification of all tumour pixels as enhancing or nonenhancing. Conventional imaging relies upon the radiologist's subjective ability to identify enhancement following intravenous administration of contrast agents. Subjective contrast enhancement is seen in almost all high grade and some low grade glioma $[10,11]$. Subtle changes, due to minimal enhancement, are open to errors of interpretation and a number of groups have described quantitative approaches. These include measurement of tumour signal intensity relative to contralateral normal-appearing white matter on either enhanced $\mathrm{T}_{1}$-weighted images $[12,13]$ or on $\mathrm{T}_{1}$ weighted images before and after intravenous administration of gadolinium $[14,15]$. Regional changes in these measurements have shown promise in early prediction of malignant transformation of low grade tumours [15] and correlate with the amount of surrounding oedema in high grade tumours [13]. An alternative approach to quantify the intensity of enhancement is to measure the concentration of contrast agent within tissue. This can be done on single enhanced images but dynamic contrast-enhanced MRI (DCE-MRI) provides additional data describing regional changes in contrast occupancy over time. The initial area under the concentration curve (IAUC) is a simple modelfree metric which has been widely used to quantify contrast enhancement in tumours on both DCE-MRI and dynamic CT $[16,17]$.

EnF can therefore be calculated by measuring the proportion of voxels within a tumour with a measurable IAUC $(>0 \mathrm{mMol} \mathrm{s})$. This simple measure identifies the presence of enhancement but does not attempt to quantify the intensity. Applying different threshold values of IAUC, above which a voxel is considered to be enhancing, will characterize the relationship between $\mathrm{EnF}$ and the intratumoural variations in the local concentration of contrast agent.

The aim of this research is to determine whether the $\mathrm{EnF}$ parameter provides any significant biological information over and above that provided by conventional microvascular biomarkers. The specific objective of this report is to provide an initial description of the behaviour of the EnF parameter in glial cell tumours where it will be affected by a wide range of physiological features such as the presence of the blood-brain barrier and variations in intracranial pressure that are not relevant in systemic tumours. We have therefore examined the relationship between $\mathrm{EnF}$ and tumour grade, described the effect of changing IAUC thresholds on EnF and compared EnF to other previously described measures for quantifying enhancement based upon signal intensity.

\section{Materials and methods}

A prospective study was performed following institutional and ethics committee review.

\section{Patients}

Patients were identified via the neuro-oncology multidisciplinary team or neurosurgical radiology meetings at Salford Royal Hospitals NHS Trust. All imaging was performed before surgery. All tumours were histologically confirmed as gliomas and were graded according to the WHO classification criteria [18]. All patients with grade IV tumours received corticosteroids treatment for a minimum of $48 \mathrm{~h}$ prior to imaging. No patients were receiving any other form of treatment at the time of imaging. All patients provided written informed consent in accordance with local research and ethics committee approval.

\section{Data acquisition}

Imaging was performed using a SENSE head coil on a 3-T Philips Achieva system (Philips Medial Systems, Best, NL) at the Translational Imaging Unit, based at Salford Royal Hospitals NHS Trust. Conventional anatomical sequences were chosen according to those used in routine clinical practice and included:

1. axial $\mathrm{T}_{1}$ inversion recovery (TR $8.4 \mathrm{~ms}$, TE $3.8 \mathrm{~ms}$, inversion time $1,150 \mathrm{~ms}$, slice thickness $1.8 \mathrm{~mm}, 256 \times$ 256),

2. axial $\mathrm{T}_{2}$ (TR $3,000 \mathrm{~ms}$, TE $80 \mathrm{~ms}$, slice thickness $3.0 \mathrm{~mm}, 1,024 \times 1,024)$,

3. coronal $\mathrm{T}_{2}$ FLAIR (TR $11,000 \mathrm{~ms}$, TE $120 \mathrm{~ms}$, inversion time $2,800 \mathrm{~ms}$, slice thickness $3.0 \mathrm{~mm}, 512 \times$ 512), and 
4. $\mathrm{T}_{1} 3 \mathrm{D}$ volume acquisition (TR $9.8 \mathrm{~ms}$, TE $4.6 \mathrm{~ms}$, slice thickness $1 \mathrm{~mm}, 256 \times 256)$ following intravenous administration of contrast medium.

Four unenhanced spoiled fast field echo $\left(\mathrm{T}_{1}\right.$-FFE spoiled gradient echo) sequences with different flip angles $\left(2^{\circ}, 5^{\circ}\right.$, $10^{\circ}, 16^{\circ}$ ) were acquired in the same geometry for calculation of baseline $\mathrm{T}_{1}$ maps (TR $3.5 \mathrm{~ms}$, TE $1.1 \mathrm{~ms}$, slice thickness $4.2 \mathrm{~mm}, 128 \times 128)$ using the standard variable flip angle relationship [19] in order to allow calculation of baseline relaxivity and thereby calculate changes in contrast concentration during the subsequent dynamic contrast-enhanced image acquisition. This was followed by a dynamic contrast-enhanced acquisition series with identical acquisition parameters as the variable flip angle baseline $T_{1}$ measurement and a $15^{\circ}$ flip angle. The series consisted of 100 volumes with temporal spacing of approximately $3.4 \mathrm{~s}$. Gadolinium-based contrast agent (Gd-DTPA-BMA; Omniscan, GE Healthcare, Amersham, UK) was injected as a bolus of dose of $0.1 \mathrm{mmol} \mathrm{kg} \mathrm{kg}^{-1}$ of body weight, at $3 \mathrm{ml} \mathrm{s}^{-1}$, after acquisition of the fifth image volume.

Data analysis

Assessment of visible enhancement on conventional imaging and tumour volumes of interest (VOIs) were defined for each tumour by an experienced neuroradiolo- gist (SJM), before histological diagnosis was confirmed. Post processing analysis was performed using in-house software (MaDyM, Manchester Dynamic Modelling). Baseline T1 images were calculated from the variable flip angle acquisitions and these values were used to calculate contrast concentration values for images in the dynamic image acquisition. Contrast concentration time course images were then used to generate parametric maps of IAUC60 (the IAUC calculated over a 60 -s period following the bolus of contrast) were produced.

EnF was calculated by dividing the volume of enhancing tissue (IAUC60 $>0 \mathrm{mMol} \mathrm{s}$ ) by the total tumour volume. Thresholded EnF curves were plotted for each tumour by calculating $\mathrm{EnF}$ at different thresholds of IAUC60. An initial analysis was performed on a subset of five grade II and ten grade IV gliomas to identify an appropriate IAUC60 threshold that provided the greatest discrimination between histological grade in this subgroup. This was done to allow division of the data into bins for statistical analysis. Mean curves were generated for the grade II and grade IV gliomas and the maximum difference between the two curves indicated the optimum threshold of IAUC60 (2.5 mMol s) for distinguishing between grades (Fig. 1).

The following metrics were calculated for each tumour: EnF where IAUC60 $>0 \mathrm{mMol} \mathrm{s}\left(\mathrm{EnF}_{\mathrm{IAUC} 60}>0\right)$, enhancing fraction where IAUC60 $>2.5 \mathrm{mMol} \mathrm{s}\left(\mathrm{EnF}_{\mathrm{IAUC} 60>2.5}\right)$, the initial gradient of the EnF/IAUC curve, $\partial \mathrm{EnF}$ (between IAUC $_{60}=0$ and $2.5 \mathrm{mMol} \mathrm{s}$ ) which represents the rate at which pixels are eliminated from the enhancing component
Fig. 1 Mean calculated enhancing fraction for different IAUC60 thresholds. Initial dataset of five grade II gliomas (triangles) and ten grade IV gliomas (circles). The error bars represent the standard error. The point of maximum difference between tumour grades was identified by subtracting the mean low grade curve from the mean high grade curve to plot a curve of difference (dashed curve). The optimised IAUC60 threshold (dashed line) was identified from this curve $(=2.5 \mathrm{mMol} \mathrm{s})$. The $\partial \mathrm{EnF}$ (dotted line) was defined as the initial average gradient of a thresholded enhancement curve between IAUC60 thresholds of $0 \mathrm{mMol} \mathrm{s}$ and $2.5 \mathrm{mMol} \mathrm{s}$

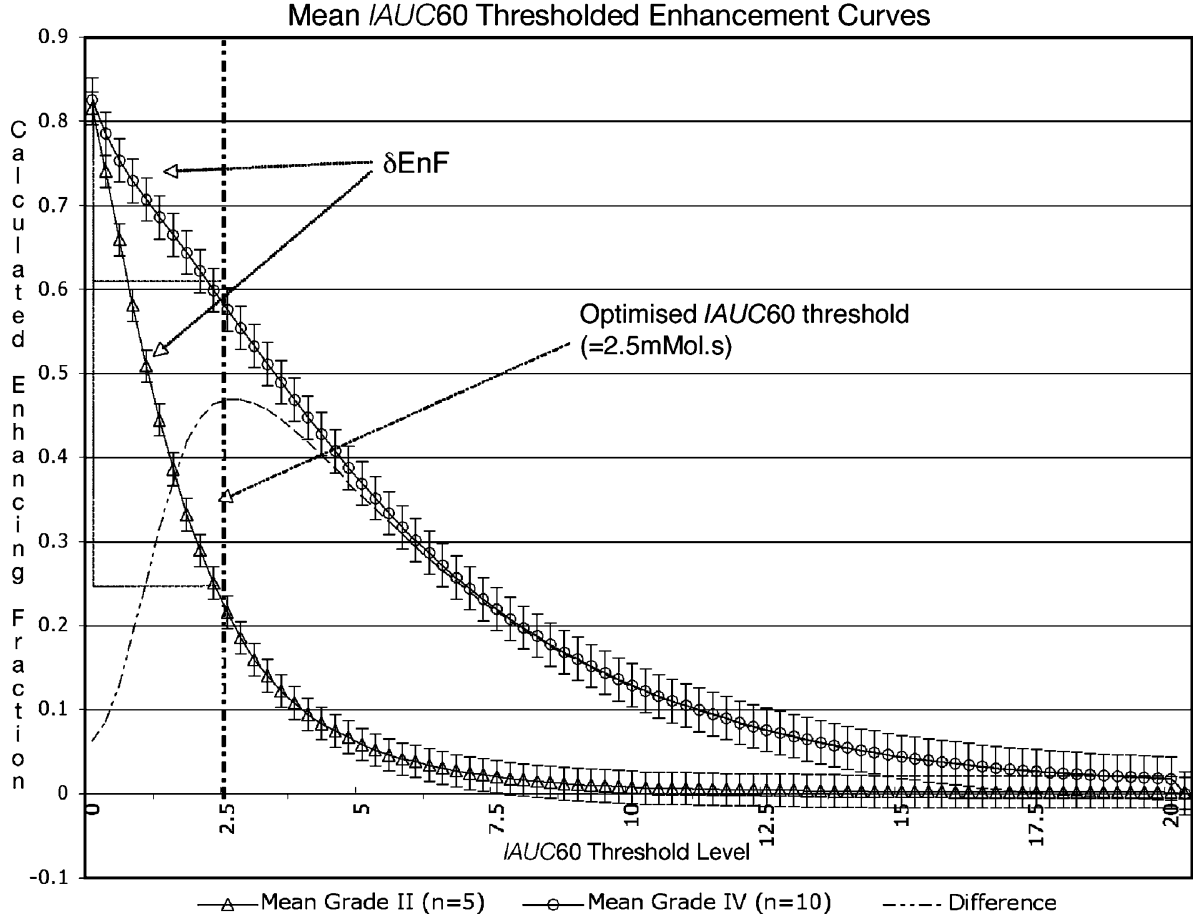


of the tumour as the threshold value of IAUC increases (Fig. 1). In addition measures of enhancement based on signal intensity were performed using methods adapted from previously described techniques by Pronin and Tofts (Appendix). Tofts' measure of enhancement, $\% E$, generates values for each voxel, the median value of $\% E$ for the whole tumour was therefore calculated to allow statistical comparison with the other measures of enhancement.

\section{Statistical analysis}

Statistical analysis was performed using SPSS (version 15.0, SPSS Inc., Chicago, USA) and MedCalc (MedCalc Software) was used for pairwise comparison of receiver operator characteristic (ROC) curves. Differences between the measured variables $\left(\mathrm{EnF}_{\text {IAUC60 }>0}, \mathrm{EnF}_{\mathrm{IAUC} 60>2.5}\right.$, $\partial \mathrm{EnF}$, median IAUC60, Pronin's degree of enhancement and median $\% E$ ) for individual tumour grades were tested using analysis of variance (ANOVA) with a posteriori pairwise testing using Tamhanes analysis (unequal variances assumed). A discriminant analysis was performed to assess the potential value of the variables in predicting histological grade, and ROC curves were generated for each variable for predicting grade II versus grade III, grade II versus grade IV, grade III versus grade IV, and low grade (II) versus high grade (III and IV) tumours.

\section{Results}

Forty-four patients (18 female; 26 male) with a mean age of 48 years (range $18-77$ years, standard deviation $=16$ years) were recruited for this study. There were 16 grade II (6 astrocytomas, 3 oligoastrocytomas and 7 oligodendrogliomas), 5 grade III (4 anaplastic astrocytomas and 1 anaplastic oligodendroglioma), and 24 grade IV gliomas (23 glioblastoma multiforme and 1 gliosarcoma); one patient had bilateral grade II tumours each of distinct histological subtype (astrocytoma and oligodendroglioma).
Fig. 2 Clustered boxplots of various enhancement measures and tumour histological grade. a IAUC60-based measures of enhancement; EnF $_{\text {IAUC60 }>0 \text {, }}$ $\mathrm{EnF}_{\text {IAUC60 }}>2.5$ and $\partial \mathrm{EnF}$. b Signal-intensity-based measures of enhancement; Pronin's degree of enhancement and Tofts' $\% E$ and $\mathbf{c}$ median IAUC60
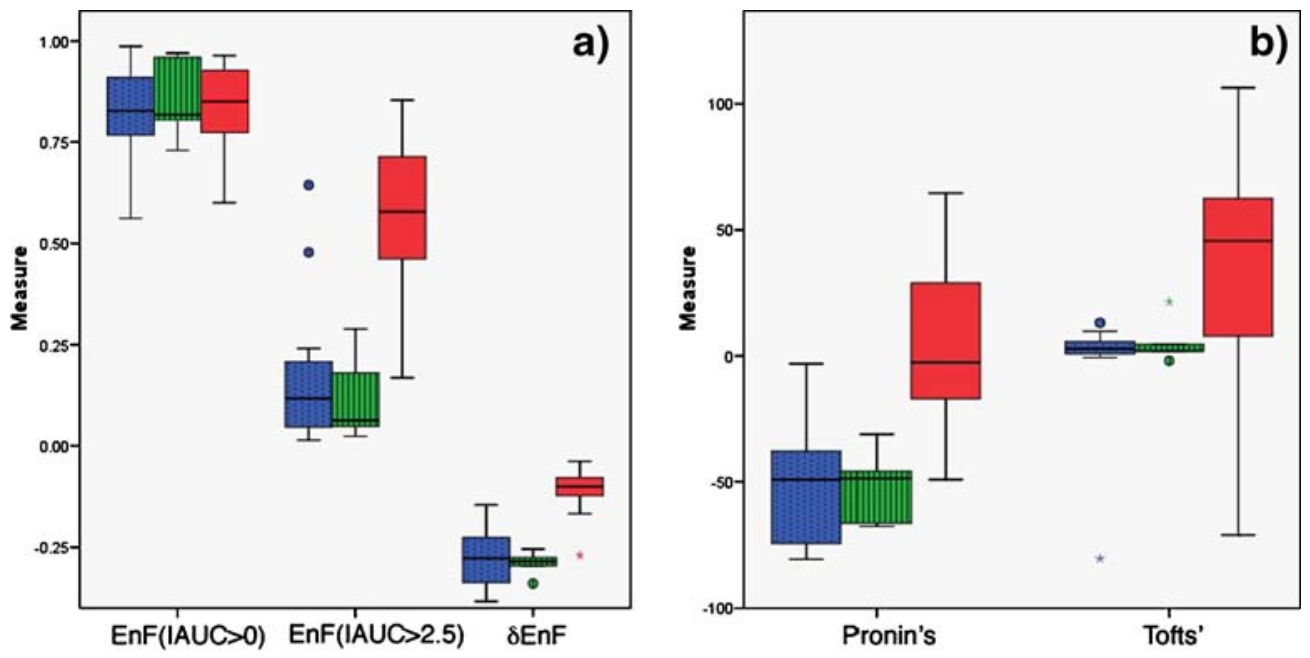

WHO Classification

回2

而3 
Five grade II tumours (1 astrocytoma, 1 oligoastrocytoma and 3 oligodendrogliomas), one grade III and all grade IV tumours demonstrated radiologically visible contrast enhancement on conventional imaging.

$\mathrm{EnF}_{\mathrm{IAUC60}>0}$ did not distinguish between individual histological grade $(p=0.894)$ or between low and high grade tumours ( $p=0.822$ ) (Fig. 2). Curves of EnF versus IAUC demonstrated clear differences between grade II and IV, grade III and IV, and low and high grade tumours, but not between grade II and III (Fig. 3). $\partial \mathrm{EnF}$ was significantly different between grade II and IV $(p<0.0005)$, as was median IAUC60 $(p<0.0005), \operatorname{EnF}_{\text {IAUC60 }}>2.5(p<0.0005)$ and both signal-intensity-based measures of enhancement (Pronin's $p<0.0005$ and Tofts' $\% E p=0.001)$. Similarly, these measures also distinguished between low and high grade tumours (RoPE $p<0.0005$, median IAUC60 $p<0.0005$, EnF $_{\text {IAUC60 }}>2.5 p<0.0005$, Pronin's $p<0.0005$ and Tofts' $\% E$ $p=0.004$ ) and between grade III and IV tumours (RoPE $p<0.0005$, median IAUC60 $p<0.0005, \quad \operatorname{EnF}_{\mathrm{IAUC}} 60>2.5$ $p<0.0005$, Pronin's $p<0.0005$ and Tofts' $\% E p=0.007$ ) (Fig. 2). No single measure distinguished grade II from grade III gliomas.

A stepwise discriminant analysis identified two canonical variables, $\partial \mathrm{EnF}$ (Wilks' lambda $=0.267, F=57.543$, $p<0.0005$ ) and Pronin's measure of enhancement (Wilks' lambda $=0.211, F=24.143, p<0.0005)$ as independent discriminants of histological grade, with RoPE accounting for $100 \%$ of the variance. The discriminant correctly classified $82.2 \%$ of all tumours, $93.8 \%$ of grade II tumours and $91.7 \%$ of grade IV tumours, but was unable to correctly classify any grade III tumour. ROC curves of all variables in predicting grade II from III, II from IV, III from IV and IV with the area under curve (AUC) measures are shown in Fig. 4. Table 1 denotes the pairwise comparisons of ROC curves for all variables in each analysis. In the analysis of grade II versus grade IV the ROC curve for $\mathrm{EnF}_{\mathrm{IAUC} 60>0}$ was significantly different, with a lower AUC, than all other variables $(p<0.001)$ and the curve for Tofts' $\% E$ was significantly different from $\partial \mathrm{EnF}(p=0.024)$. Comparison of low versus high grade showed only the variable EnF $_{\text {IAUC60>0 }}$ to differ significantly from all other variables.

There were insufficient numbers for formal statistical analysis of patients based on histological subtype of grade II and III tumours, although a tendency was seen for increasing $\mathrm{EnF}_{\mathrm{IAUC60>0}}$ in tumours with an oligodendroglial component (Fig. 5).

\section{Discussion}

This study was designed to examine the behaviour of EnF in cerebral tumours. The EnF has been proposed as a simple measure of bulk tumour perfusion [3, 4]. EnF has been shown to predict outcome to first-line chemotherapy
Fig. 3 Mean thresholded enhancement curves for all grades of tumour. Clear differences were seen between grade II and IV tumours, but not grade II and III. Grade II glioma (triangles, $n=16$ ), grade III glioma (crosses, $n=5$ ) and grade IV glioma (circles, $n=24$ ). Error bars were of a similar magnitude to those in Fig. 1 but have been excluded for improved visualisation of the separate grade II and III curves

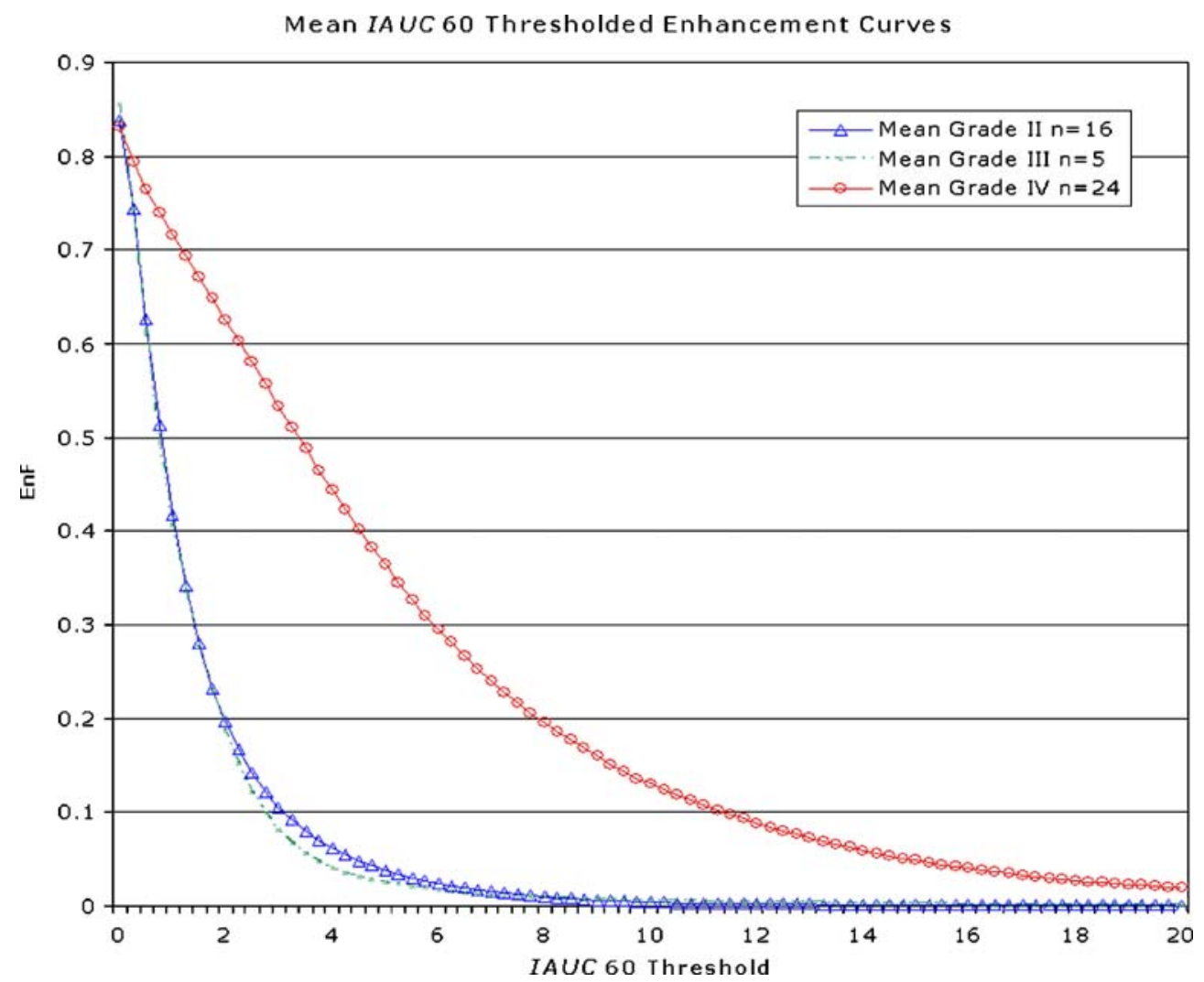


Fig. 4 ROC curves for $\partial \mathrm{EnF}$ and Pronin's measure of enhancement as predictors of tumour grade: a grade II versus grade III gliomas, $\mathbf{b}$ grade II versus grade IV gliomas, $\mathbf{c}$ grade III versus grade IV gliomas and d high (grades III and IV) versus low (grade II) glioma
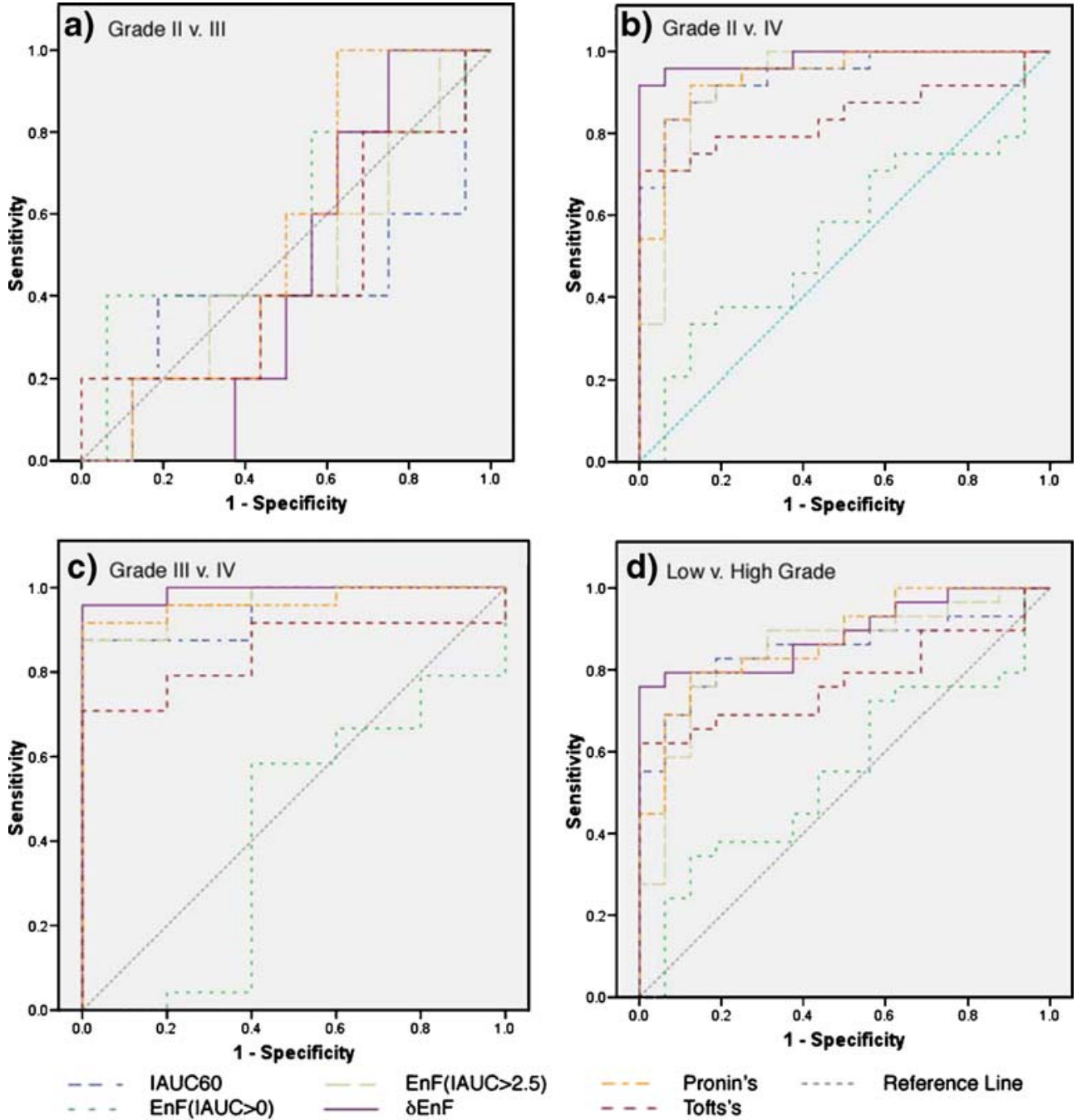

in patients with advanced ovarian cancer [5] where nonenhancement principally reflected areas of tumour necrosis. EnF has also been shown to change rapidly in response to drug-induced variations in tumour interstitial pressure [3] where nonenhancement represents failure of perfusion of viable vessels. The EnF has not been examined in cerebral tumours, where enhancement characteristics are affected by a number of unique physiological mechanisms: specifically the presence of the blood-brain barrier and disease-related variations in intracranial pressure. Although we have used the relationship between tumour grade and $\mathrm{EnF}$ to begin to characterize this biomarker it is important to state that we are attempting to validate $\mathrm{EnF}$ as a biomarker and are not proposing it as a method for clinical tumour grading at this stage.

Interestingly, we found that $\mathrm{EnF}_{\mathrm{IAUC60}>0}$ provided no discrimination between low and high grade tumours since small degrees of contrast enhancement were detectable in large portions of low grade tumours despite subjective lack of enhancement. This is counterintuitive since low grade tumours are commonly described as "nonenhancing" based on subjective criteria. However, low grade tumours are perfused and therefore contain small amounts of contrast within the vascular space even where the blood-brain barrier is intact, although IAUC60 values are expected to be low. In high grade tumours higher values of IAUC60 are expected since vascular fractions are far higher and breakdown of the blood brain-barrier leads to extravasation of contrast medium. This is supported by the results of this study where median values of IAUC60 were significantly higher in grade IV (mean $=4.43+$ $2.23 \mathrm{mMol} \mathrm{s})$ than grade II (mean $=1.23+0.79 \mathrm{mMol} \mathrm{s})$ tumours.

The relationship between EnF and IAUC will reflect a combination of processes. In enhancing portions of a tumour the amount of contrast observed within a voxel will depend on blood flow, vascular fraction, endothelial permeability and the size of the extravascular extracellular space. These relationships are well understood and can be described by a series of widely employed pharmacokinetic 
Table 1 Pairwise comparison of ROC curves

\begin{tabular}{|c|c|c|c|c|c|}
\hline & & Grade II versus III & Grade II versus IV & Grade III versus IV & Low versus high grade \\
\hline \multicolumn{6}{|l|}{ Area under curve (AUC) } \\
\hline \multicolumn{2}{|l|}{$\mathrm{EnF}_{\mathrm{IAUC} 60>0}$} & 0.563 & 0.549 & 0.417 & 0.552 \\
\hline \multicolumn{2}{|l|}{$\mathrm{EnF}_{\mathrm{IAUC} 60>2.5}$} & 0.463 & 0.924 & 0.967 & 0.845 \\
\hline \multicolumn{2}{|l|}{ IAUC60 } & 0.413 & 0.94 & 0.95 & 0.849 \\
\hline \multicolumn{2}{|l|}{ Pronin's } & 0.538 & 0.94 & 0.967 & 0.871 \\
\hline \multicolumn{2}{|l|}{$\partial \mathrm{EnF}$} & 0.438 & 0.982 & 0.992 & 0.888 \\
\hline \multicolumn{2}{|l|}{ Tofts' } & 0.45 & 0.841 & 0.85 & 0.774 \\
\hline \multicolumn{6}{|c|}{ Pairwise comparisons of AUC ( $p$ values) } \\
\hline \multirow[t]{5}{*}{ EnF $_{\text {IAUC60>0 }}$ versus } & $\mathrm{EnF}_{\mathrm{IAUC} 60>2.5}$ & 0.823 & $<0.001 *$ & $0.002 *$ & $<0.001 *$ \\
\hline & IAUC60 & 0.824 & $<0.001 *$ & $0.002 *$ & $<0.001^{*}$ \\
\hline & Pronin's & 0.85 & $<0.001^{*}$ & $0.004 *$ & $0.001 *$ \\
\hline & $\partial \mathrm{EnF}$ & 1 & $<0.001^{*}$ & $0.003^{*}$ & $<0.001^{*}$ \\
\hline & Tofts' & 0.898 & $<0.001^{*}$ & $0.016^{*}$ & $0.006^{*}$ \\
\hline \multirow[t]{4}{*}{ EnFF $_{\text {IAUC60 }>2.5}$ versus } & IAUC60 & 0.251 & 0.442 & 0.28 & 0.853 \\
\hline & Pronin's & 1 & 0.759 & 1 & 0.682 \\
\hline & $\partial \mathrm{EnF}$ & 0.863 & 0.149 & 0.329 & 0.424 \\
\hline & Tofts' & 0.92 & 0.14 & 0.072 & 0.249 \\
\hline \multirow[t]{3}{*}{ IAUC60 versus } & Pronin's & 0.756 & 1 & 0.645 & 0.734 \\
\hline & $\partial \mathrm{EnF}$ & 0.867 & 0.242 & 0.202 & 0.472 \\
\hline & Tofts' & 0.787 & 0.095 & 0.113 & 0.262 \\
\hline \multirow[t]{2}{*}{ Pronin's versus } & $\partial \mathrm{EnF}$ & 0.872 & 0.308 & 0.396 & 0.778 \\
\hline & Tofts' & 0.932 & 0.066 & 0.062 & 0.104 \\
\hline$\partial \mathrm{EnF}$ versus & Tofts' & 0.936 & $0.024 *$ & 0.052 & 0.121 \\
\hline
\end{tabular}

$\partial \mathrm{EnF}$ has the largest AUC for differentiating grade II from IV, grade III from IV and low from high grade tumours $A U C$ area under curve

*Significance taken as $p<0.05$

models [20]. Pixels will be classified as nonenhancing when the voxel contains too little contrast media to provide a degree of enhancement sufficient to exceed the threshold set for tissue classification. This may occur in necrotic tumour or in perfused tumour where the blood-brain barrier is intact or where flow is absent or considerably reduced.

The observations presented here demonstrate a clear and significant difference in the relationship between EnF and IAUC in low and high grade tumours. Increasing the threshold of IAUC60 above which a voxel is considered to enhance caused the calculated enhancing fraction to fall, as the proportion of "enhancing" voxels declines. In low grade tumours increasing the IAUC60 threshold caused a steep drop-off in EnF. In the grade IV tumours many voxels contain a high concentration of contrast agent so that increasing the IAUC60 threshold results in a more gradual decline in EnF. These observations also indicate that the use of a single definition of enhancement such as those used in previous studies of systemic tumours $[3,5]$ may be misleading and obscure information of potential biological importance. We have therefore described two novel metrics that characterize the relationship between EnF and IAUC: $\partial \mathrm{EnF}$ and $\mathrm{EnF}_{\mathrm{IAUC}}>2.5 . \partial \mathrm{EnF}$ demonstrated excellent discrimination, with high sensitivity $(93.7 \%)$ and specificity (95.8\%), between grade II and grade IV tumours, and between grade III and IV tumours (sensitivity 95.83\%, specificity $100 \%$; Fig. 4). $\partial \mathrm{EnF}$ also demonstrated the highest AUC values of all variables on ROC analysis (these differed significantly from $\mathrm{EnF}_{\mathrm{IAUC} 60>0}$ and Tofts' $\% E$ on comparison of grade II versus IV and $\mathrm{EnF}_{\mathrm{IAUC60}>0}$ alone for low versus high grade tumours). Both signal-intensity based measures of enhancement also demonstrated significant differences between grade II and IV tumours, although considerable overlap was seen between high and low grade tumours (Fig. 2). In addition, Pronin's measure of enhancement discriminated between grade III and IV tumours; however, this did not differ significantly from $\partial \mathrm{EnF}$ on ROC analysis.

Identification of grade III tumours by imaging remains a significant problem. Clinically they are treated as malignant dedifferentiated tumours although their prognosis is 




Fig. 5 Scatter plots of En FIAUC60>0 $_{\text {in relation to tumour histological }}$ subtype for grade II and III gliomas. $A$ astrocytoma, $O A$ oligoastrocytoma, $O$ oligodendroglioma, $A A$ anaplastic astrocytoma, $A O$ anaplastic oligodendroglioma. There was a tendency for En $n_{\text {FIAUC60 }>2.5}$ to increase in the presence of oligodendroglial component in both grade II and grade III tumours, with higher En $_{\text {FIAUC60 }>0}$ in oligoastrocytomas (squares, $n=3$ ) and oligoden-

better than glioblastoma. Any classification system would therefore ideally identify them as a separate subgroup but should at least not group them together with grade 2 tumours. Unfortunately many studies have been unable to separate grade II and grade III tumours on the basis of imaging biomarkers. White et al. studied 24 oligodendrogliomas (16 grade II and 8 grade III) by using a signalintensity-based measure of enhancement and found no difference between grade II and grade III tumours [14]. The current study also failed to differentiate between these two groups. Other studies have been able to differentiate grade III from grade II but not from grade IV [9]. The development of imaging biomarkers to accurately identify grade III tumours is hampered by considerable variability in the accuracy of pathological classification and by the frequent occurrence of grade II tumours with an oligodendroglial component which are known to be relatively vascular [21]. This was the case in the current study as $62 \%$ of low grade tumours had significant oligodendroglioma components. In addition we had only five grade III tumours so that we can draw no conclusions concerning the accurate grading of this group.

One potential weakness of the current study is the use of an IAUC threshold derived from a small subgroup of the study population. However, we are not proposing this threshold for clinical use but only to support binning of the datasets in this study for statistical comparison. The study is also limited by the relatively small numbers (only 5 grade III gliomas) and by failure to separate tumours into histological subtypes. drogliomas (triangles, $n=7$ ) than in astrocytomas (diamonds, $n=6$ ) and higher in the anaplastic astrocytomas (crosses, $n=4)$ than the anaplastic oligodendroglioma (scored-through cross, $n=1$ ). In addition, there was a suggestion that the anaplastic astrocytomas had a higher En FIAUC60>0 $_{\text {than }}$ the astrocytomas and the anaplastic oligodendroglioma had a higher $E_{\text {FIAUC60 }>0}$ than the oligodendrogliomas

In conclusion, metrics based on the enhancing tumour fraction show significant differences between low and high grade glioma. Their use as a tool for clinical grading is limited by failure to differentiate grade II and grade III tumours. However, as we stated above, the aim of this study was to provide an initial description of the behaviour of enhancing-fraction-based metrics in intracerebral tumours and not to produce a clinical grading tool. The $\partial \mathrm{EnF}$ metric incorporates components of both $\mathrm{EnF}$ and quantitative enhancement behaviour. This relationship is likely to be affected by factors that will not be reflected in more conventional enhancement metrics, particularly tumour interstitial and intracranial pressure. Although further work is clearly required to further characterise and validate these metrics as potential biomarkers the characteristics demonstrated here make them of potential interest for the monitoring of low grade glioma for the detection of malignant transformation and for detection of changes in hydrodynamic equilibrium that occur with tumour growth and in response to novel antivascular treatments in high grade glioma.

\section{Conclusion}

We have described two new biomarkers ( $\partial \mathrm{EnF}$ and EnF $_{\text {IAUC }>2.5}$ ) which characterize the relationship between the intensity of tumour enhancement and enhancing fraction. These markers are simple to derive and provide excellent discrimination between grade II and IV cerebral 
gliomas. We believe that these findings justify further research to evaluate these potential biomarkers and assess their role in monitoring treatment response and predicting outcome and early malignant transformation.

Acknowledgements This work from funded by a Research Training Bursary from Cancer Research UK, ref: C21247/A6840. $\mathrm{S}$. Mills is funded by a Cancer Research UK Clinicians Training Fellowship ref: C21247/A7473. J. O'Connor is funded by a Cancer Research UK Clinicians Training Fellowship ref: C19221/A6086. G. Buonaccorsi is funded on a Cancer Research UK Program Grant ref: C237/A6295. C. Rose is funded by GlaxoSmithKline.

Open Access This article is distributed under the terms of the Creative Commons Attribution Noncommercial License which permits any noncommercial use, distribution, and reproduction in any medium, provided the original author(s) and source are credited.

\section{Appendix}

Both Pronin's and Tofts measures of enhancement were calculated using modified techniques based on the original literature $[5,6,8]$. Analysis was performed in MATLAB (The MathWorks Inc., Natick, Massachusetts) using the pre- and post-contrast $\mathrm{T}_{1}$-weighted images through the lesion. As a result of the sagittal oblique acquisition of the images, contralateral normal-appearing white matter (NAWM) could not be assessed and the analysis was, therefore, based upon remote NAWM. An area of remote NAWM was identified distinct and separate from the tumour and a VOI was defined. The same tumour VOI used in the perfusion analysis was used in the calculation of signal-intensity-based enhancement.

Pronin's measure of the degree of enhancement $[5,6]$ :

$=100 \times\left(\frac{S_{\text {tumourVOI }+C}-S_{\mathrm{NAWMVOI}+\mathrm{C}}}{S_{\mathrm{NAWMVOI}+\mathrm{C}}}\right)$

where:

$S_{\text {tumourVOI }+ \text { C }}=$ mean post-contrast signal intensity for whole tumour volume of interest

$S_{\mathrm{NAWMVOI}+\mathrm{C}}=$ mean post-contrast signal intensity for whole remote NAWM volume of interest

This produced a measure of enhancement for the whole tumour VOI.

Tofts' measure of enhancement $(\% E)[8]$ :

$=100 \times\left(\frac{S_{\text {tumour }+C}-S_{\text {tumour }}}{S_{N A W M}}\right)$

where:

$S_{\text {tumour }+\mathrm{C}}=$ signal intensity for tumour voxel post-contrast

$S_{\text {tumour }}=$ signal intensity for tumour voxel pre-contrast

$S_{\mathrm{NAWM}}=$ mean signal intensity for remote NAWM on both pre- and post-contrast imaging

This produced a measure of enhancement for each voxel within the tumour VOI. To allow comparison with other measures of enhancement median values were calculated.

\section{References}

1. Lopes MBS (2005) Neuropathology. In: Schiff D, O’Neill BP (eds) Principles of neuro-oncology. McGraw-Hill, New York, pp 81-120

2. Winkler F, Kozin SV, Tong RT et al (2004) Kinetics of vascular normalization by VEGFR2 blockade governs brain tumor response to radiation: role of oxygenation, angiopoietin-1, and matrix metalloproteinases. Cancer Cell 6:553-563

3. Jayson GC, Parker GJ, Mullamitha S et al (2005) Blockade of platelet-derived growth factor receptor-beta by CDP860, a humanized, PEGylated diFab', leads to fluid accumulation and is associated with increased tumor vascularized volume. J Clin Oncol 23:973981
4. Mullamitha SA, Ton NC, Parker GJ et al (2007) Phase I evaluation of a fully human anti-alphav integrin monoclonal antibody (CNTO 95) in patients with advanced solid tumors. Clin Cancer Res 13:2128-2135

5. O'Connor JP, Jayson GC, Jackson A et al (2007) Enhancing fraction predicts clinical outcome following first-line chemotherapy in patients with epithelial ovarian carcinoma. Clin Cancer Res 13:6130 6135

6. Jackson A (2004) Analysis of dynamic contrast enhanced MRI. Br J Radiol 77 (Spec No 2):S154-S166

7. Aronen HJ, Gazit IE, Louis DN et al (1994) Cerebral blood volume maps of gliomas: comparison with tumor grade and histologic findings. Radiology 191:41-51
8. Haroon HA, Patankar TA, Dow G et al (2002) Relationship between vasculoar endothelial permeability and histological grade in human gliomas using a novel first pass model. In: Proceedings of the 10th international meeting of the nternational Society for Magnetic Resonance in Medicine, Honolulu, Hawaii, May 2002, p 2113

9. Patankar TF, Haroon HA, Mills SJ et al (2005) Is volume transfer coefficient (K (trans)) related to histologic grade in human gliomas? AJNR Am J Neuroradiol 26:2455-2465

10. Barker FG 2nd, Chang SM, Huhn SL et al (1997) Age and the risk of anaplasia in magnetic resonance-nonenhancing supratentorial cerebral tumors. Cancer 80:936-941 
11. Hammoud MA, Sawaya R, Shi W et al (1996) Prognostic significance of preoperative MRI scans in glioblastoma multiforme. J Neurooncol 27:65-73

12. Pronin IN, Holodny AI, Kornienko VN et al (1997) The use of hyperventilation in contrast-enhanced MR of brain tumors. AJNR Am J Neuroradiol 18:1705-1708

13. Pronin IN, Holodny AI, Petraikin AV (1997) MRI of high-grade glial tumors: correlation between the degree of contrast enhancement and the volume of surrounding edema. Neuroradiology 39:348-350
14. White ML, Zhang Y, Kirby P et al (2005) Can tumor contrast enhancement be used as a criterion for differentiating tumor grades of oligodendrogliomas? AJNR Am J Neuroradiol 26:784-790

15. Tofts PS, Benton CE, Weil RS et al (2007) Quantitative analysis of wholetumor Gd enhancement histograms predicts malignant transformation in low-grade gliomas. J Magn Reson Imaging 25:208-214

16. Evelhoch JL (1999) Key factors in the acquisition of contrast kinetic data for oncology. J Magn Reson Imaging $10: 254-259$

17. Parker GJM, Buckley DL (2005) Tracer kinetic modelling for T1-weighted DCE-MRI. In: Jackson A, Buckley DL, Parker GJM (eds) Dynamic contrastenhanced magnetic resonance imaging in oncology. Springer, Berlin, pp 81-92

18. Louis DN, Ohgaki H, Wiestler OD et al (2007) The 2007 WHO classification of tumours of the central nervous system. Acta Neuropathol (Berl) 114:97-109
19. Haase A (1990) Snapshot FLASH MRI. Applications to T1, T2, and chemical-shift imaging. Magn Reson Med 13:77-89

20. Buckley DL, Parker GJM (2005) Measuring contrast agent concentration in $\mathrm{T}_{1}$-weighted dynamic contrast enhanced MRI. In: Jackson A, Buckley DL, Parker GJM (eds) Dynamic contrast-enhanced magnetic resonance imaging in oncology. Springer, Berlin, p 69

21. Cha S, Lupo JM, Chen MH et al (2007) Differentiation of glioblastoma multiforme and single brain metastasis by peak height and percentage of signal intensity recovery derived from dynamic susceptibility-weighted contrastenhanced perfusion MR imaging. AJNR Am J Neuroradiol 28:10781084 